\title{
Targeted therapy of ovarian cancer including immune check point inhibitor
}

\author{
Jin Young $\mathrm{Kim}^{1,2}$, Chi Heum $\mathrm{Cho}^{3}$, and Hong Suk Song ${ }^{1}$
}

\begin{abstract}
${ }^{1}$ Division of Hematology/Oncology, Department of Internal Medicine, Keimyung University Dongsan Medical Center, Daegu; ${ }^{2}$ Pain Research Center, Keimyung University School of Medicine, Daegu; ${ }^{3}$ Department of Obstetrics and Gynecology, Keimyung University Dongsan Medical Center, Daegu, Korea
\end{abstract}

Received: December 28, 2016 Accepted: January 3, 2017

\section{Correspondence to}

Jin Young Kim, M.D.

Division of Hematology/

Oncology, Department of

Internal Medicine, Keimyung

University Dongsan Medical

Center, 56 Dalseong-ro, Jung-gu,

Daegu 41931, Korea

Tel: $+82-53-250-7476$

Fax: +82-53-425-6476

E-mail: takgu@dsmc.or.kr
Epithelial ovarian cancer is the eighth most common cause of cancer-related deaths in women because most patients present with advanced stage disease at the time of diagnosis. Although cytoreductive surgery and platinum-based chemotherapy remain the gold standards of treatment, the recurrence rate of ovarian cancer remains high. Attempts to improve this standard two-drug chemotherapy by adding a third cytotoxic drug have failed to affect either progression-free survival or overall survival and have resulted in an increase in toxic side effects. Some anti-angiogenic agents, poly(ADP-ribose) polymerase, and immune checkpoint inhibitors have shown efficacy in early stages of development for the treatment of epithelial ovarian cancer. As demonstrated in recent clinical trials, the use of bevacizumab, cediranib, pazopanib, olaparib, and rucaparib, either alone or in combination with conventional cytotoxic agents, improves progression-free survival. Trials on immune checkpoint inhibitors such as nivolumab have revealed prolonged responses in a small set of ovarian cancer cases but require further exploration. In this review, we discuss the role of targeted therapies against ovarian cancer, including the use of immune checkpoint inhibitors.

Keywords: Ovarian neoplasms; Molecular targeted therapy; Immunotherapy

\section{INTRODUCTION}

Epithelial ovarian cancer (EOC) is the eighth most common cause of cancer-related deaths in Korean women, with an estimated 1,021 deaths occurring annually nationwide in 2014 [1]. For the past decade, the standard treatment for women with advanced ovarian cancer has been optimal cytoreductive surgery and platinum-based chemotherapy. Although approximately $80 \%$ of patients respond to first-line chemotherapy, more than $70 \%$ of patients with advanced stage disease recur within 5 years and develop drug resistance [2,3]. Attempts to improve standard two-drug chemotherapy by adding a third cytotoxic drug have failed to affect either progression-free survival(PFS) or overall survival(OS) and have resulted in an increase in toxic side effects [4-7]. Advances in tradi- tional cytotoxic chemotherapies, such as intraperitoneal administration and dose-dense therapeutic regimens, are improving response rates, as are novel agents such as bevacizumab or poly(ADP-ribose) polymerase (PARP) inhibitors $[8,9]$. Unfortunately, platinum-refractory advanced ovarian cancer does not show a proper response rate; therefore, more effective treatment strategies, particularly molecular targeting agents, are required to improve survival rates for patients with advanced ovarian cancer.

\section{CURRENT TREATMENT MODALITIES}

Optimal cytoreductive surgery is effective in most patients with ovarian cancer; however, in cases of advanced 
Table 1. Summary of efficacy data from randomized, controlled phase III trials of bevacizumab in advanced ovarian cancer

\begin{tabular}{|c|c|c|c|c|}
\hline Study & Regimen & ORR (CR + PR), \% & Median PFS, mon & Median OS, mon \\
\hline $\begin{array}{l}\text { GOG-0218 [21] } \\
(\mathrm{n}=1,873)\end{array}$ & $\begin{array}{l}\mathrm{CP}+\text { placebo vs. } \mathrm{CP}+\mathrm{Bev} \\
\text { vs. } \mathrm{CP}+\mathrm{Bev} \rightarrow \mathrm{Bev} \\
\text { maintenance }\end{array}$ & - & $\begin{array}{c}10.3 \text { vs. } 11.2 \text { vs. } 14.1 \\
(\mathrm{HR}, 0.908 ; p=0.16)^{\mathrm{a}} \\
(\mathrm{HR}, 0.717 ; p<0.001)^{\mathrm{b}}\end{array}$ & $\begin{array}{l}39.3 \text { vs. } 38.7 \text { vs. } 39.7 \\
(H R, 1.036 ; p=0.76)^{\mathrm{a}} \\
(\mathrm{HR}, 0.915 ; p=0.45)^{\mathrm{b}}\end{array}$ \\
\hline $\begin{array}{l}\text { ICON } 7[22,23] \\
(n=1,528)\end{array}$ & $\begin{array}{l}\mathrm{CP} \text { vs. } \mathrm{CP}+\mathrm{Bev} \rightarrow \mathrm{Bev} \\
\text { maintenance }\end{array}$ & $\begin{array}{c}48 \text { vs. } 67 \\
(p<0.001)\end{array}$ & $\begin{array}{c}17.4 \text { vs. } 19.8 \\
(\mathrm{HR}, 0.87 ; p=0.04)\end{array}$ & 44.6 vs. 44.5 \\
\hline $\begin{array}{l}\text { OCEANS }[24,27] \\
(n=484)\end{array}$ & $\begin{array}{l}\mathrm{CG}+\text { placebo vs. } \mathrm{CG}+ \\
\mathrm{Bev}\end{array}$ & $\begin{array}{l}57.4 \text { vs. } 78.5 \\
(p<0.0001)\end{array}$ & $\begin{array}{c}8.4 \text { vs. } 12.4 \\
(\mathrm{HR}, 0.484 ; p<0.0001)\end{array}$ & $\begin{array}{c}33.6 \mathrm{c} \mathrm{vs} .32 .9^{c} \\
(\mathrm{HR}, 0.960 ; p=0.736)\end{array}$ \\
\hline $\begin{array}{l}\text { AURELIA }[28,29] \\
(\mathrm{n}=361)\end{array}$ & $\begin{array}{l}\text { CTx (PLD, P, or Top) vs. } \\
\text { CTx }+ \text { Bev }\end{array}$ & $\begin{array}{c}11.8 \text { vs. } 27.3 \\
(p=0.001)\end{array}$ & $\begin{array}{c}3.4 \text { vs. } 6.7 \\
(\mathrm{HR}, 0.48 ; p<0.001)\end{array}$ & $\begin{array}{c}13.3 \text { vs. } 16.6 \\
(\mathrm{HR}, 0.85 ; p=0.174)\end{array}$ \\
\hline
\end{tabular}

ORR, overall response rate; CR, complete response; PR, partial response; PFS, progression-free survival; OS, overall survival; GOG, Gynecologic Oncology Group; C, carboplatin; P, paclitaxel; Bev, bevacizumab; HR, hazard ratio; ICON, International Collaborative Ovarian Neoplasm; OCEANS, platinum-sensitive recurrent disease; G, gemcitabine; AURELIA, platinum-resistant ovarian cancer; CTx, chemotherapy; PLD, pegylated liposomal doxorubicin; Top, topotecan.

${ }^{\mathrm{a}} \mathrm{CP}+\mathrm{Bev}$ vs. $\mathrm{CP}+$ placebo.

${ }^{\mathrm{b}} \mathrm{CP}+\mathrm{Bev} \rightarrow$ Bev vs. $\mathrm{CP}+$ placebo.

${ }^{\mathrm{c}}$ Interim data.

stage ovarian cancer (International Federation Gynecology Obstetrics [FIGO] stage IIB-IIIC), platinum-based combination chemotherapy is recommended [10]. A modified carboplatin plus paclitaxel regimen with weekly paclitaxel results in improved long-term outcomes compared to the 3-weekly regimen in a phase III study on advanced ovarian cancer [11-14]. More than $80 \%$ of patients respond to first-line chemotherapy; however, approximately $70 \%$ of patients with advanced EOC recur within 5 years and develop drug resistance $[2,3]$.

For recurrent EOC, there is no evidence of a survival benefit following early treatment of relapse on the basis of a raised cancer antigen 125 (CA-125) concentration alone [15]. The treatment options for recurrent EOC are based on the timing and nature of the recurrence and the extent of prior chemotherapy [16]. Patients responding to front-line platinum-based chemotherapy are very likely to respond to a rechallenge with platinum-based chemotherapy. However, patients relapsing after front-line platinum/paclitaxel chemotherapy have more side effects, especially neurotoxicity. Refractory ovarian cancer occurs in patients who have failed to achieve a response to therapy. Patients with platinum-resistant ovarian cancer do not respond to initial therapy or their disease recurs after a short (<6 months) treatment-free interval [17]. The response rate in patients with platinum refractory status is below $10 \%$ [18]. Due to the number and diversity of ac- tive agents presently available for second-line treatment of ovarian cancer (i.e., pegylated liposomal doxorubicin, docetaxel, topotecan, vinorelbine, and belotecan), clinicians and patients often consider treatments beyond the second-line setting, assuming adequate organ function and overall performance status [17].

\section{TARGETED THERAPIES}

Vascular endothelial growth factor (VEGF) expression is higher in advanced stage tumors compared to those at an early stage [19]. Angiogenesis plays an integral role in the initiation and progression of ovarian carcinogenesis [20]. Given the association between increased angiogenesis and the progression of ovarian cancer, a number of anti-angiogenic agents are currently in development as potential treatment options for patients with advanced disease.

\section{Bevacizumab}

Proper efficacy data is currently available from four important phase III randomized trials in advanced ovarian cancer (Table 1). The first two trials tested bevacizumab in combination with carboplatin/paclitaxel in an adjuvant setting: the International Collaborative Ovarian Neoplasm (ICON-7) trial and the Gynecologic Oncol- 
ogy Group (GOG) trial 218 [21-23]. The other two trials evaluated bevacizumab in recurrent cases of ovarian cancer: an ovarian cancer study comparing the efficacy and safety of chemotherapy and anti-angiogenic therapy in platinum-sensitive recurrent disease (OCEANS) and bevacizumab use in platinum-resistant ovarian cancer (AURELIA) [24-26].

ICON-7 enrolled 1,528 patients, $70 \%$ of whom had stage IIIc or stage IV ovarian cancer. At a median follow-up time of 36 months, patients in the bevacizumab arm showed a significant improvement in median PFS (2 months). The maximal effect of this trial was observed at 12 months but decreased after 24 months. A recently updated analysis showed similar PFS and OS benefits in the bevacizumab group [23].

GOG protocol 218 was a three-arm placebo-controlled study. In the standard treatment arm, patients were given carboplatin (area under the curve [AUC] 5 or 6 ) and paclitaxel $\left(175 \mathrm{mg} / \mathrm{m}^{2}\right)$ every 3 weeks for six cycles. In the bevacizumab throughout arm, bevacizumab was given with chemotherapy for two to six cycles and then continued every 3 weeks for a total of 22 cycles. In the bevacizumab initiation arm, bevacizumab was given with chemotherapy for two to six cycles and then continued with placebo in cycles seven to 22. The dose of bevacizumab given intravenously $(15 \mathrm{mg} / \mathrm{kg}$ ) was double the dose given in ICON-7. The improvement in median PFS was significant in the bevacizumab throughout arm, but there was no significant difference in OS between the three arms (Table 1) [21-24,27-29].

The OCEANS trial was a randomized, multi-center, blinded, placebo-controlled phase III trial. Patients were randomly assigned to carboplatin plus gemcitabine combined with bevacizumab or placebo for six to 10 cycles. Bevacizumab or placebo was continued until disease progression. PFS for the bevacizumab arm was superior to that for the placebo arm (12.4 months vs. 8.4 months, respectively). In addition, bevacizumab therapy caused a significant improvement in the objective response rate (78.5\% vs. $57.4 \%$, respectively) and duration of response (10.4 months vs. 7.4 months, respectively). There was no OS benefit for patients who received bevacizumab compared to the placebo arm (33.6 months vs. 32.9 months, respectively) [24].

The AURELIA trial was the first randomized phase III trial to evaluate bevacizumab in combination with che- motherapy in platinum-resistant ovarian cancer [25,26]. Pegylated liposomal doxorubicin $\left(40 \mathrm{mg} / \mathrm{m}^{2}\right)$ was given on day 1 every 4 weeks; weekly paclitaxel $\left(80 \mathrm{mg} / \mathrm{m}^{2}\right)$ was administered on days $1,8,15$, and 22 every 4 weeks; or topotecan $\left(4 \mathrm{mg} / \mathrm{m}^{2}\right)$ was administered on days 1,8 , and 15 every 4 weeks or topotecan $\left(1.25 \mathrm{mg} / \mathrm{m}^{2}\right)$ was given on days 1 through 5 every 3 weeks. Bevacizumab (10 mg/kg every 2 weeks or $15 \mathrm{mg} / \mathrm{kg}$ every 3 weeks) was given until progression, unacceptable toxicity, or consent withdrawal. There was a 3-month prolongation of PFS with the addition of bevacizumab. The difference in OS was not significant (Table 1), but the overall response rate (ORR) was higher in the bevacizumab arm compared to without bevacizumab (11.8\% vs. $27.3 \%$, respectively).

\section{Pazopanib}

Pazopanib is an oral multi-target tyrosine kinase inhibitor (TKI) of vascular endothelial growth factor receptor (VEGFR)-1, -2, and -3, platelet-derived growth factor receptor (PDGFR)- $\alpha$ and $-\beta$, and $c$ kit. A phase II open-label study evaluated oral pazopanib monotherapy in patients with low-volume recurrent ovarian cancer with complete CA-125 response to initial platinum-based chemotherapy and subsequent elevation of CA-125. Patients were treated with pazopanib (80o mg once daily) until progressive disease or unacceptable toxicity. The ORR was $18 \%$ in patients with measurable disease at baseline [30]. The international Arbeitsgemeinschaft Gynaekologische Onkologie Studiengruppe Ovarialkarzinom trial 16 (AGOOVAR 16) was a phase III randomized control trial that evaluated the role of pazopanib in maintenance therapy of ovarian cancer FIGO stages II-IV with no evidence of progression after primary therapy, consisting of surgery and at least five cycles of platinum/taxane chemotherapy; patients were randomized 1:1 to receive pazopanib (80o mg once per day) or placebo for up to 24 months. Maintenance pazopanib prolonged PFS compared to placebo (17.9 months vs. 12.3 months, respectively). Pazopanib maintenance therapy provided a median improvement of 5.6 months in PFS in patients with advanced ovarian cancer who did not progress after first-line chemotherapy. OS data did not suggest any benefit. Grade 3 or 4 adverse events of hypertension (30.8\%), neutropenia (9.9\%), liver-related toxicity ( $9.4 \%)$, diarrhea (8.2\%), fatigue (2.7\%), thrombocytopenia (2.5\%), and palmar-plantar erythrodysesthesia 
(1.9\%) were significantly higher in the pazopanib arm. Treatment discontinuation related to adverse events was higher among patients treated with pazopanib (33.3\%) compared to placebo (5.6\%) [31].

\section{Cediranib}

Cediranib is an oral TKI of VEGFR-1, -2 , and -3 , and c-kit. Matulonis et al. [32] conducted a phase II study on cediranib for recurrent ovarian cancer. Cediranib was administered as a daily oral dose; the original dose was $45 \mathrm{mg}$ and was lowered to $30 \mathrm{mg}$ due to toxicity. The clinical benefit rate (complete response or partial response, stable disease > 16 weeks, or CA-125 non-progression $>16$ weeks) was $30 \%$. Eleven patients (23\%) were removed from the study because of toxicity before two cycles. Grade 3 toxicities (> 20\% of patients) included hypertension (46\%), fatigue (24\%), and diarrhea (13\%) [32]. Cediranib was the first VEGFR TKI to show an OS benefit when used as maintenance therapy in recurrent ovarian cancer; this was demonstrated in the ICON-6 trial. The ICON-6 trial is a three-arm, three-stage, double-blind, placebo-controlled randomized trial in first relapse of platinum-sensitive ovarian cancer. Patients were randomized (2:3:3) to receive six cycles of carboplatin (AUC 5 or 6) plus paclitaxel $\left(175 \mathrm{mg} / \mathrm{m}^{2}\right)$ with either placebo, cediranib (20 mg per day) followed by placebo, or cediranib (20 mg per day) followed by cediranib. Cediranib or placebo was continued for 18 months or until disease progression. The cediranib maintenance arm showed improvements in PFS and OS by 2.7 months. Although more adverse effects occurred in the cediranib arm, these were found to be tolerable [33].

\section{PARP INHIBITORS}

\section{Olaparib}

The first clinical illustration of synthetic lethality in cancer was from the development of PARP inhibitors for the treatment of cancers with defects in the BRCA1 (BReast CAncer gene 1) or BRCA2 tumor suppressor proteins, which are involved in the repair of DNA damage. Although this is a promising approach, multiple potential resistance mechanisms have been identified [34]. Ledermann et al. [35] conducted a randomized, double-blind, placebo-controlled phase II trial to evaluate maintenance treatment with olaparib in patients with platinum-sensitive, relapsed, high-grade serous ovarian cancer who received two or more platinum-based regimens and had a partial or complete response to a platinum-containing regimen. Patients received olapar$\mathrm{ib}$ (400 mg twice daily) or placebo. PFS was significantly longer with olaparib than with placebo (8.4 months vs. 4.8 months, respectively) [35]. According to the retrospective analysis of the phase II trial in advanced ovarian cancer with BRCA1/2 mutations, there was a significantly prolonged PFS in the olaparib group compared to the placebo group (11.2 months vs. 4.3 months, respectively). There was also a strong trend towards an OS advantage, although this was not statistically significant (34.9 months vs. 31.9 months, respectively). The OS benefit could not be shown because most patients received other post-progression therapies and lived for many more years. The most common grade 3 or worse adverse events in the olaparib group were fatigue ( $7 \%$ vs. $3 \%$, respectively) and anemia (5\% vs. $<1 \%$, respectively) [36].

Two ongoing multi-center double-blind randomized phase III trials are Studies of Olaparib in Ovarian Cancer (SOLO) 1 and 2. These studies focus on the role of olaparib in the maintenance of high-grade ovarian cancer in patients with the BRCA mutation. SOLO 1 includes patients with newly diagnosed advanced ovarian cancer who have responded to first-line platinum therapy and SOLO 2 includes cases of recurrent ovarian cancer who have completed more than two lines of platinum therapy. Clinicians expect the maturated data from two important trials.

\section{Rucaparib}

Rucaparib is a potent oral inhibitor of PARP 1 and 2 according to Assessment of Rucaparib in Ovarian Cancer Trial 2 (ARIEL 2), which enrolled patients with platinum-sensitive recurrent EOC with the BRCA mutation. ARIEL 2 prospectively tested a novel next-generation sequencing-based homologous recombination deficiency (HRD) assay and algorithm to predict rucaparib sensitivity by assessing tumor BRCA status and genome-wide loss of heterozygosity (LOH). The patients received rucaparib (600 mg twice daily) in three pre-defined HRD subgroups: tumor BRCA ${ }^{\text {mut }}, \mathrm{BRCA}^{\mathrm{wt}} / \mathrm{LOH}^{\text {high }}$, and $\mathrm{BRCA}^{\mathrm{WT}} / \mathrm{LOH}^{\text {low }}$. Efficacy data indicated ORRs of $69 \%$, $39 \%$, and $11 \%$, respectively. Responses occurred in both 
germline $\mathrm{BRCA}^{\mathrm{mut}}(14 / 19,74 \%)$ and somatic $\mathrm{BRCA}^{\mathrm{mut}}$ (10/16, 63\%) tumors [37]. The pivotal ARIEL 3 study compared the effects of rucaparib versus placebo.

\section{PARP inhibitor in combination with anti-angiogenic drugs}

The combination of olaparib and cediranib versus single-agent olaparib in a phase II study on patients with platinum-sensitive recurrent ovarian cancer showed better PFS in the combination arm compared to the single-agent olaparib arm (17.7 months vs. 9.0 months, respectively). A significant improvement in PFS occurred in germline BRCA wild-type patients receiving cediranib/olaparib. Grade 3 and 4 adverse events, including fatigue, diarrhea, and hypertension, were more common in patients who received cediranib/olaparib therapy [38].

\section{IMMUNOTHERAPIES}

The use of monoclonal antibody-based checkpoint blockade for the treatment of ovarian cancer is also being developed, as well as targeting cytotoxic T-lymphocyte associated protein 4 (CTLA- 4 ) or the anti-programmed cell death ligand-1/programmed cell death-1 (PD-L1/PD1) axis. The anti-CTLA-4 antibody being widely tested is ipilimumab. Hodi and colleagues [39] provided the first indication that this drug may be useful; they enrolled two ovarian cancer patients in an early trial and demonstrated a reduction in CA-125 in one patient and stabilization in another. PD-1 is a co-inhibitory receptor that is expressed on activated $\mathrm{T}$ cells and regulates anti-tumor immunity. Nivolumab is a fully humanized immunoglobulin $\mathrm{G}_{4}$ that blocks the engagement of PD-1 by PD-1 ligands. Nivolumab was administered every 2 weeks to patients with advanced or relapsed platinum-resistant ovarian cancer. Fifteen patients were treated with nivolumab ( $1 \mathrm{mg} / \mathrm{kg}, \mathrm{n}=10 ; 3 \mathrm{mg} / \mathrm{kg}, \mathrm{n}=5)$ and evaluated. The $1 \mathrm{mg} / \mathrm{kg}$ treatment cohort showed a $20 \%$ partial response rate and a 50\% disease control rate, and tolerated the side effects well [40]. The expression of PD-L1 in the tumor microenvironment appears to be crucial for therapeutic activity, and initial trials have suggested that positive PD-L1 tumor expression is associated with higher response rates. However, subsequent observations have questioned the prospect of using PD-L1 as a biomarker for selecting patients for therapy, especially since many patients considered PD-L1-negative benefit from treatment. However, there is no definitive test for determining PD-Li expression, and a cut-off reference for PD-L1-positive status has not yet been established.

\section{CONCLUSIONS}

Ovarian cancer remains the most challenging cancer to clinicians even though treatment options have improved over several decades. These improvements have relied on optimal surgery and platinum-based chemotherapies. Recently, the most considerable improvement in ovarian cancer treatment came from bevacizumab and PARP inhibitor therapies. Although ovarian cancer is a targetable tumor, its biology is unique and highly heterogeneous. Newer developed drugs, especially the PD-1/PD-L1 antibody, have shown promising results; however, we do not have a proper prognostic marker. Further research is required to understand the molecular mechanisms more fully and to develop targeting combination therapies to overcome resistance, which may help conquer this disease with minimal toxicity. More tailored treatments based on molecular characteristics are expected in the near future.

\section{Conflict of interest}

No potential conflict of interest relevant to this article was reported.

\section{REFERENCES}

1. National Cancer Information Center. Percentage of deaths from 10 cancer deaths among men in 2015 [Internet]. Goyang (KR): National Cancer Information Center, c2012 [cited 2017 Aug 3]. Available from: http://www.cancer. go.kr/mbs/cancer/subview.jsp?id=cancer_040202000000.

2. Heintz AP, Odicino F, Maisonneuve P, et al. Carcinoma of the ovary: FIGO 26th Annual Report on the Results of Treatment in Gynecological Cancer. Int J Gynaecol Obstet 2006;95 Suppl 1:S161-S192.

3. Monk BJ, Coleman RL. Changing the paradigm in the treatment of platinum-sensitive recurrent ovarian cancer: from platinum doublets to nonplatinum doublets and 
adding antiangiogenesis compounds. Int J Gynecol Cancer 2009;19 Suppl 2:S63-S67.

4. Bookman MA, Brady MF, McGuire WP, et al. Evaluation of new platinum-based treatment regimens in advanced-stage ovarian cancer: a Phase III Trial of the Gynecologic Cancer Intergroup. J Clin Oncol 2009;27:14191425 .

5. du Bois A, Weber B, Rochon J, et al. Addition of epirubicin as a third drug to carboplatin-paclitaxel in first-line treatment of advanced ovarian cancer: a prospectively randomized gynecologic cancer intergroup trial by the Arbeitsgemeinschaft Gynaekologische Onkologie Ovarian Cancer Study Group and the Groupe d'Investigateurs Nationaux pour l'Etude des Cancers Ovariens. J Clin Oncol 2006;24:1127-1135.

6. Pfisterer J, Weber B, Reuss A, et al. Randomized phase III trial of topotecan following carboplatin and paclitaxel in first-line treatment of advanced ovarian cancer: a gynecologic cancer intergroup trial of the AGO-OVAR and GINECO. J Natl Cancer Inst 2006;98:1036-1045.

7. Hoskins P, Vergote I, Cervantes A, et al. Advanced ovarian cancer: phase III randomized study of sequential cisplatin-topotecan and carboplatin-paclitaxel vs carboplatin-paclitaxel. J Natl Cancer Inst 2010;102:1547-1556.

8. Armstrong DK, Bundy B, Wenzel L, et al. Intraperitoneal cisplatin and paclitaxel in ovarian cancer. N Engl J Med 2006;354:34-43.

9. Meehan RS, Chen AP. New treatment option for ovarian cancer: PARP inhibitors. Gynecol Oncol Res Pract 2016;3:3.

10. Colombo N, Peiretti M, Parma G, et al. Newly diagnosed and relapsed epithelial ovarian carcinoma: ESMO Clinical Practice Guidelines for diagnosis, treatment and follow-up. Ann Oncol 2010;21 Suppl 5:v23-v30.

11. Katsumata N, Yasuda M, Takahashi F, et al. Dose-dense paclitaxel once a week in combination with carboplatin every 3 weeks for advanced ovarian cancer: a phase 3, open-label, randomised controlled trial. Lancet 2009;374:1331-1338.

12. Katsumata N, Yasuda M, Isonishi S, et al. Long-term results of dose-dense paclitaxel and carboplatin versus conventional paclitaxel and carboplatin for treatment of advanced epithelial ovarian, fallopian tube, or primary peritoneal cancer (JGOG 3016): a randomised, controlled, open-label trial. Lancet Oncol 2013;14:1020-1026.

13. Pignata S, Scambia G, Katsaros D, et al. Carboplatin plus paclitaxel once a week versus every 3 weeks in patients with advanced ovarian cancer (MITO-7): a randomised, multicentre, open-label, phase 3 trial. Lancet Oncol 2014;15:396-405.

14. Chan JK, Brady MF, Penson RT, et al. Weekly vs. every3-week paclitaxel and carboplatin for ovarian cancer. $\mathrm{N}$ Engl J Med 2016;374:738-748.

15. Rustin GJ, van der Burg ME, Griffin CL, et al. Early versus delayed treatment of relapsed ovarian cancer (MRC OVo5/ EORTC 55955): a randomised trial. Lancet 2010;376:11551163.

16. National Comprehensive Cancer Network. Ovarian cancer [Internet]. Fort Washington (PA): National Comprehensive Cancer Network, c2017 [cited 2017 Aug 3]. Available from: https://www.nccn.org.

17. Markman M, Bookman MA. Second-line treatment of ovarian cancer. Oncologist 2000;5:26-35.

18. Markman M, Rothman R, Hakes T, et al. Second-line platinum therapy in patients with ovarian cancer previously treated with cisplatin. J Clin Oncol 1991;9:389-393.

19. Lokadasan R, James FV, Narayanan G, Prabhakaran PK. Targeted agents in epithelial ovarian cancer: review on emerging therapies and future developments. Ecancermedicalscience 2016;10:626.

20. Abu-Jawdeh GM, Faix JD, Niloff J, et al. Strong expression of vascular permeability factor (vascular endothelial growth factor) and its receptors in ovarian borderline and malignant neoplasms. Lab Invest 1996;74:1105-1115.

21. Burger RA, Brady MF, Bookman MA, et al. Incorporation of bevacizumab in the primary treatment of ovarian cancer. N Engl J Med 2011;365:2473-2483.

22. Perren TJ, Swart AM, Pfisterer J, et al. A phase 3 trial of bevacizumab in ovarian cancer. N Engl J Med 2011;365:24842496.

23. Oza AM, Cook AD, Pfisterer J, et al. Standard chemotherapy with or without bevacizumab for women with newly diagnosed ovarian cancer ( $\mathrm{ICON} 7)$ : overall survival results of a phase 3 randomised trial. Lancet Oncol 2015;16:928936.

24. Aghajanian C, Blank SV, Goff BA, et al. OCEANS: a randomized, double-blind, placebo-controlled phase III trial of chemotherapy with or without bevacizumab in patients with platinum-sensitive recurrent epithelial ovarian, primary peritoneal, or fallopian tube cancer. J Clin Oncol 2012;30:2039-2045.

25. Pujade-Lauraine E, Hilpert F, Weber B, et al. Bevacizum- 
ab combined with chemotherapy for platinum-resistant recurrent ovarian cancer: the AURELIA open-label randomized phase III trial. J Clin Oncol 2014;32:1302-1308.

26. Stockler MR, Hilpert F, Friedlander M, et al. Patient-reported outcome results from the open-label phase III AURELIA trial evaluating bevacizumab-containing therapy for platinum-resistant ovarian cancer. J Clin Oncol 2014;32:1309-1316.

27. Aghajanian C, Nycum L, Goff B, et al. Updated overall survival analysis in OCEANS, a randomized phase III trial of gemcitabine (G) + carboplatin (C) and bevacizumab (BV) or placebo (PL) followed by BV or PL in platinum-sensitive recurrent epithelial ovarian (ROC), primary peritoneal (PPC), or fallopian tube cancer (FTC). Proceedings of the 37th Congress of the European Society for Medical Oncology (ESMO); 2012 Sep 28-Oct 2; Vienna (AT). Austria: Oxford University Press, 2012: 319.

28. Pujade-Lauraine E, Hilpert F, Weber B, et al. AURELIA: a randomized phase III trial evaluating bevacizumab (BEV) plus chemotherapy (CT) for platinum (PT)-resistant recurrent ovarian cancer (OC). J Clin Oncol 2012;30(18 Suppl):327s.

29. Witteveen P, Lortholary A, Fehm T, et al. Final overall survival (OS) results from AURELIA, an open-label randomised phase III trial of chemotherapy (CT) with or without bevacizumab (BEV) for platinum-resistant recurrent ovarian cancer (OC). Eur J Cancer 2013;49 Suppl 3:S3.

30. Friedlander M, Hancock KC, Rischin D, et al. A phase II, open-label study evaluating pazopanib in patients with recurrent ovarian cancer. Gynecol Oncol 2010;119:32-37.

31. du Bois A, Floquet A, Kim JW, et al. Incorporation of pazopanib in maintenance therapy of ovarian cancer. J Clin Oncol 2014;32:3374-3382.

32. Matulonis UA, Berlin S, Ivy $\mathrm{P}$, et al. Cediranib, an oral inhibitor of vascular endothelial growth factor receptor kinases, is an active drug in recurrent epithelial ovarian, fallopian tube, and peritoneal cancer. J Clin Oncol 2009;27:5601-5606.

33. Raja FA, Griffin CL, Qian W, et al. Initial toxicity assessment of ICON6: a randomised trial of cediranib plus chemotherapy in platinum-sensitive relapsed ovarian cancer. Br J Cancer 2011;105:884-889.

34. Lord CJ, Ashworth A. Mechanisms of resistance to therapies targeting BRCA-mutant cancers. Nat Med 2013;19:1381-1388.

35. Ledermann J, Harter P, Gourley C, et al. Olaparib maintenance therapy in platinum-sensitive relapsed ovarian cancer. N Engl J Med 2012;366:1382-1392.

36. Ledermann J, Harter P, Gourley C, et al. Olaparib maintenance therapy in patients with platinum-sensitive relapsed serous ovarian cancer: a preplanned retrospective analysis of outcomes by BRCA status in a randomized phase 2 trial. Lancet Oncol 2014;15:852-861.

37. McNeish IA, Oza AM, Coleman RL, et al. Results of ARIEL2: a phase 2 trial to prospectively identify ovarian cancer patients likely to respond to rucaparib using tumor genetic analysis. J Clin Oncol 2015;33(15 Suppl):5508.

38. Liu JF, Barry WT, Birrer M, et al. Combination cediranib and olaparib versus olaparib alone for women with recurrent platinum-sensitive ovarian cancer: a randomized phase 2 study. Lancet Oncol 2014;15:1207-1214.

39. Hodi FS, Mihm MC, Soiffer RJ, et al. Biologic activity of cytotoxic $\mathrm{T}$ lymphocyte-associated antigen 4 antibody blockade in previously vaccinated metastatic melanoma and ovarian carcinoma patients. Proc Natl Acad Sci U S A 2003;100:4712-4717.

40. Hamanishi J, Mandai M, Ikeda T, et al. Efficacy and safety of anti-PD-1 antibody (Nivolumab: BMS-936558, ONO4538 ) in patients with platinum-resistant ovarian cancer. J Clin Oncol 2014;32(15 Suppl):5511. 